\title{
Outcomes of TB treatment in HIV co-infected TB patients in Ethiopia: a cross-sectional analytic study
}

\author{
Solomon Ahmed Ali ${ }^{1,4^{*}}$, Thandisizwe R. Mavundla ${ }^{1}$, Ribka Fantu ${ }^{2}$ and Tadesse Awoke ${ }^{3}$
}

\begin{abstract}
Background: TB and HIV are the most prevalent communicable diseases of major public health importance in the populations of sub-Saharan African countries, and an estimated $30 \%$ of HIV infected persons have dual infection with TB. TB is the leading cause of death in HIV infected individuals, and HIV co-infected TB patients have multiple individual, disease specific and treatment related factors that can adversely affect their treatment outcomes. There is lack of evidence on the individual patient outcomes of HIV co-infected TB patients who receive anti-TB treatment. It is relevant to understand the differential patient outcomes of HIV co-infected TB patients and identify the factors that are associated with these outcomes.
\end{abstract}

Methods: A comparative analysis was done on the data from a random sample of 575 TB patients who were enrolled for TB treatment from January 2013 to December 2013 at eight health facilities in Ethiopia. A descriptive analysis was done on the data, and chi-square test and logistic regression analysis was conducted to compare TB treatment outcomes based on HIV status and to identify factors associated with these outcomes.

Results: Out of a total of 575 TB patients enrolled into the study, 360 (62.6\%) were non-HIV infected, 169 (29.4\%) were HIV co-infected, and 46 (8\%) had no documented HIV status. The overall treatment success rate was $91.5 \%$ for all the study participants. HIV co-infected TB patients have a treatment success rate of $88.2 \%$ compared with $93.6 \%$ for non-HIV infected study participants $(P=0.03)$. HIV co-infected TB patients had a significantly higher rate $(11.8 \%$ versus $6.4 \%, P=0.03)$ of unfavourable outcomes. The cure rate was significantly lower $(10.1 \%$ versus $24.2 \%$, $P=0.001)$ and the death rate higher in HIV co-infected TB patients ( $8.3 \%$ versus $2.5 \%, P=0.014$ ). Age and TB classification were significantly associated with treatment outcome. No association was found with starting ART, Cotrimoxazole prophylactic treatment or enrolment in HIV care.

Conclusions: There is high TB treatment success rate among patients who have been treated for TB, but the treatment success rate and the cure rate in HIV co-infected TB patients is lower than that observed in non-HIV infected patients. Patients with advanced age and those with smear positive pulmonary TB have unfavourable treatment outcomes.

Keywords: TB treatment outcome, TB/HIV co-infection, Treatment success rate, Favourable outcomes, Unfavourable outcomes, Cure rate, Death rate

\footnotetext{
* Correspondence: hnb2@cdc.gov

'Department of Health Studies, University of South Africa (UNISA), Pretoria,

South Africa

${ }^{4}$ Addis Ababa, Ethiopia

Full list of author information is available at the end of the article
} 


\section{Background}

Tuberculosis (TB) and Human Immunodeficiency Virus (HIV) are the most prevalent communicable diseases of major public health concern in the populations of subSaharan African (SSA) countries including Ethiopia. Co-infection with HIV and TB is very common with an estimated $30 \%$ of HIV infected persons having dual infection with TB ([1]: [S202-203]). About $80 \%$ of the total estimated disease burden of HIV associated TB is found in countries of SSA, and this part of the world has the "highest rates of cases and deaths per capita" attributable to TB disease [2]. TB is among the leading causes of morbidity and mortality in HIV infected individuals; and in patients with TB disease, co-infection with HIV significantly complicates both the diagnosis and management of TB disease ([1] [S202-203]).

Many of the countries most severely affected by the two epidemics are resource poor settings with weak health systems ([3]:S1-S3). Decreasing the burden of disease from both infections among the population and improving the health outcomes of the individual patients attending medical services is of paramount importance to mitigate the adverse impact from these conditions.

Early detection of TB disease and prompt initiation of effective treatment with potent anti-TB drug regimens is required to decrease morbidity and mortality from $\mathrm{TB}$ disease in HIV infected patients [4]. Furthermore, this approach is critically important for prevention of transmission of TB within the population.

Co-infection with HIV is associated with significantly increased likelihood of mortality from TB disease, and HIV co-infected TB patients have significantly lower cure rates and lower treatment success rates compared to nonHIV infected TB patients ([5]:222-226; [6]). HIV patients with active TB disease have a probability of dying of $15-20 \%$ at 1 year while those without active TB disease have 7-8 \% probability of dying at 1 year ([7]:1605-1612).

Ethiopia is one of the countries in SSA that is hardest hit by the TB and HIV epidemics. With an estimated national adult prevalence of $1.5 \%$, it is estimated that, in 2013 , there were 734,048 adults and children infected with HIV in Ethiopia [8]. The incidence of TB was 258 cases per 100,000 populations in 2011 [2]. The prevalence of HIV among incident TB cases was found to be $17 \%$ [2]. This indicates that there is a high burden from each disease and a considerable co-infection rate. However, there is lack of evidence on the individual patient outcomes of co-infected patients with active TB disease and receiving anti-TB treatment in Ethiopia. It is essential and timely to better understand how and why HIV co-infected TB patients have unfavourable outcomes, and comparison of the outcomes of co-infected TB patients with those without HIV infection is relevant to understand the magnitude of the problem.
TB/HIV co-infected patients have multiple individual, disease specific and treatment related factors that can adversely affect their treatment outcomes [9]. Identifying these factors would help to provide better medical care to these patients for improved health outcomes.

The objective of this study was to assess and explain the outcomes of TB treatment in TB patients with respect to their HIV status, and to identify the associated underlying factors that contribute to the occurrence of these outcomes.

\section{Methods}

This study used cross-sectional study design.

The target population for this study was all patients diagnosed with active TB disease and put on anti-TB treatment regimens. The accessible population was all patients diagnosed with active $\mathrm{TB}$ disease and put on anti-TB treatment regimens in the selected health facilities for this study.

The data source for this study was the TB register at the TB clinics of the health facilities where all patients diagnosed with active TB disease are put on anti-TB treatment regimens and monitored throughout the course of their treatment. The TB registers in the TB clinics of these health facilities are used to record all relevant patient level and clinical information for the treatment and monitoring of TB patients, and also for reporting of patient level data. Therefore, the sampling frame for this study is the list of all TB patients enrolled (within the selected time frame for the study, which is January 2013-December 2013) in the selected health facilities at which the study is conducted.

\section{Operational definitions}

([10]:52; [11]:43; [12]).

\section{TB infection}

Infection with mycobacterium tuberculosis bacilli.

\section{Active TB disease}

Presence of signs and symptoms of TB disease in an individual who is infected with mycobacterium tuberculosis bacilli.

\section{Case of tuberculosis}

A definite case of TB (a pulmonary TB case with one or more initial sputum smear examinations positive for acid-fast bacilli) or one in which a health worker (clinician or other medical practitioner) has diagnosed TB and has decided to treat the patient with a full course of TB treatment.

\section{HIV infection}

Infection with the Human Immune-deficiency Virus (HIV) that is confirmed by approved serologic tests. 


\section{TB/HIV co-infection}

The presence of both TB and HIV infection in an individual patient.

\section{TB treatment outcome}

The final known status of a TB patient who was started on anti-TB treatment.

\section{Cured}

An initially smear-positive patient who is sputum smearnegative at, or 1 'month' prior to, the completion of TB treatment and on at least one previous occasion (usually at the end of the $2^{\text {nd }}$ or $5^{\text {th }}$ month).

\section{Treatment completed}

A patient who completed anti-TB treatment without evidence of failure but for whom sputum smear or culture results are not available in the last month of treatment and on at least one previous occasion.

\section{Treatment failure}

A patient whose sputum smear or culture is positive at 5 months or later during treatment. Also included in this definition are patients found to harbour a multidrugresistant (MDR) strain at any point of time during the treatment, whether they are smear-negative or -positive.

\section{Defaulter}

A patient who has been on treatment for at least 4 weeks and whose treatment was interrupted for 8 or more consecutive weeks.

\section{Died}

A patient who dies for any reason during the course of treatment.

\section{Transfer out}

A patient who started treatment and has been transferred to another reporting unit and for whom the treatment outcome is not known at the time of evaluation of treatment results.

\section{Treatment success}

The sum of patients who are declared 'cured' and those who have 'completed' treatment.

\section{Inclusion criteria}

- Any individual who has been diagnosed with active TB disease based on the Ethiopian national TB guidelines recommendations

- He or she has been started on a course of anti-TB treatment regimen within the time frame of the study period

\section{Exclusion criteria}

- Any individual who has taken less than 4 weeks of the course of anti-TB treatment regimen

- Individuals less than 15 years of age at initiation of anti-TB treatment

The study sites (health facilities) included in this study are selected based on convenience (geographic accessibility) and patient load among all health centres within the Addis Ababa city administration which is a major urban setting and the capital city of Ethiopia. Based on the current TB patient load at each site and the number of patients required to meet the sample size, eight health facilities are selected for conducting the study.

\section{Sampling}

A simple random sampling method was used to enrol eligible study participants into the study. The number of study participants to be enrolled from each selected health facility was determined proportionally (based on patient load). A table of random numbers was used to select and enrol study participants into the study sample from the sampling frame at each health facility.

\section{Ethical issues related to sampling and data management} Prior to the commencement of the study, the final version of the study protocol had been submitted and ethical clearance obtained from the local IRB (Addis Ababa Regional Health Bureau Ethical Review Committee) and the UNISA College of Human Sciences (CHS) Health Studies Higher Degrees Committee (HSHDC).

\section{Sample size}

The sample size calculation was done using the Epi Info 7 statistical software program. The prevalence of HIV in incident TB cases in Ethiopia was estimated to be $17 \%$ [2]. The reported TB treatment success rate for year 2009 indicated in the $2011 \mathrm{WHO}$ global TB report [6] shows a TB treatment success rate of 72 and $88 \%$ for HIV co-infected and non-HIV infected TB patients respectively. Using these background data as inputs to the epi info 7 statistical software to calculate the sample size, 350 TB patients are needed (61 HIV co-infected, 289 non HIV infected) with a two sided confidence level of $95 \%$ and a power of $80 \%$.

\section{Data management and analysis}

A paper based data collection tool was used to abstract data from the TB registers. The collected data were transferred to an electronic format (Excel sheet) that was prepared to capture the study variables from the data collection tool. The data in the Excel electronic format were cleaned (See 'Additional file 1' for supporting 
data). Study participants without documented TB treatment outcome data $(<1 \%$ of the sample) were excluded from the study sample as their data cannot be part of the analysis. There was very limited information on patients who declined to be tested for HIV. These study participants with missing HIV status information were kept with the study sample to be part of the data analysis.

The investigator imported the cleaned data to SPSS statistical software (PASW Statistics 18) from the electronic Excel format. The continuous variable age was recoded into age groups for the purpose of the analysis. The treatment outcome was also recoded into two groups. The treatment outcomes 'Cured' and 'Treatment completed' were grouped together as favourable treatment outcomes or treatment success. The other treatment outcomes were put together as unfavourable treatment outcomes.

\section{Results}

\section{Sample characteristics}

The age of the study participants ranged from 15 to 90 years with $58.3 \%$ in the $25-49$ age range (Table 1), and male gender accounted for more than half of the study sample.

Among the 575 study participants enrolled in the study, 529 (92\%) of them had a documented HIV test offered and performed at or prior to the beginning of

Table 1 Distribution of characteristics among study participants $(N=575)$

\begin{tabular}{lll}
\hline & Number & Percent \\
\hline Age group (years) & 151 & 26.3 \\
$15-24$ & 184 & 32.0 \\
$25-34$ & 151 & 26.3 \\
$35-49$ & 60 & 10.4 \\
$50-64$ & 29 & 5.0 \\
$64-90$ & 575 & 100.0 \\
Total & & \\
HIV status & 360 & 62.6 \\
Non-reactive (HIV-negative) & 169 & 29.4 \\
Reactive (HIV-positive) & 46 & 8.0 \\
Missing (not done) & 575 & 100.0 \\
Total & & \\
Category of TB treatment & 2 & 0.3 \\
Default & 1 & 0.2 \\
Failure & 488 & 84.9 \\
New & 51 & 8.9 \\
Other & 17 & 3.0 \\
Relapse & 16 & 2.8 \\
Transfer-in & 575 & \\
Total & & \\
\hline
\end{tabular}

their course of anti-TB treatment, and almost one-third of the study participants have documented HIV coinfection (i.e. HIV positive) as shown in Table 1.

Most of the study participants were diagnosed with pulmonary tuberculosis $(55.8 \%)$ and a quarter of all cases of TB were found to have smear-positive pulmonary tuberculosis. The majority of the study participants were new TB patients who started their TB treatment at the health facility where they have completed their course of TB treatment (Table 1).

More than $90 \%$ of the study participants with TB/ HIV co-infection were enrolled in HIV care, and most of them provided with Anti-Retroviral Treatment (ART) and/or co-trimoxazole prophylactic treatment (Fig. 1, $N=169$ ).

\section{Treatment outcomes of the study participants}

Analysis of the study data demonstrates that the outcome of TB treatment for the study participants shows a very high overall treatment success rate $(91.5 \%)$ defined as either 'Cured' or 'Treatment completed' after a course of anti-TB treatment (Fig. 2, $N=575$ ). The treatment success rate was $93.6 \%$ among non-HIV infected TB patients in contrast to $88.2 \%$ among HIV coinfected TB patients (Table 2) which was statistically significant $(P=0.03$, Chi-Square test).

\section{TB treatment outcomes in relation to HIV status}

In this study, HIV co-infected TB patients have a treatment success rate of $88.2 \%$, and a higher rate $(11.8 \%)$ of unfavourable treatment outcomes (defined as death, default or failure) compared to those without underlying HIV infection (6.4 \%) as shown in Table 2. These differences in treatment outcomes were statistically significant $(P=0.03$, Chi-Square test). Figure 3 shows the comparison of all the TB treatment outcomes in relation to HIV status.

The cure rate among HIV co-infected TB patients was significantly lower and the death rate significantly higher (Table 3) than that among those without underlying HIV infection (10.1 \% versus $24.2 \%$ and $8.3 \%$ vs $2.5 \%$, respectively).

Other characteristics associated with treatment outcome Further analysis of the study data was performed to identify other factors (variables) that potentially affect the outcome of TB treatment in the study population. This analysis showed that age and TB classification are significantly associated with $\mathrm{TB}$ treatment outcome. Table 4 provides a detailed account of the results of the analysis. No association was observed between TB treatment outcome and receiving other medical care services like starting ART, co-trimoxazole prophylactic treatment or enrolment in HIV care. 


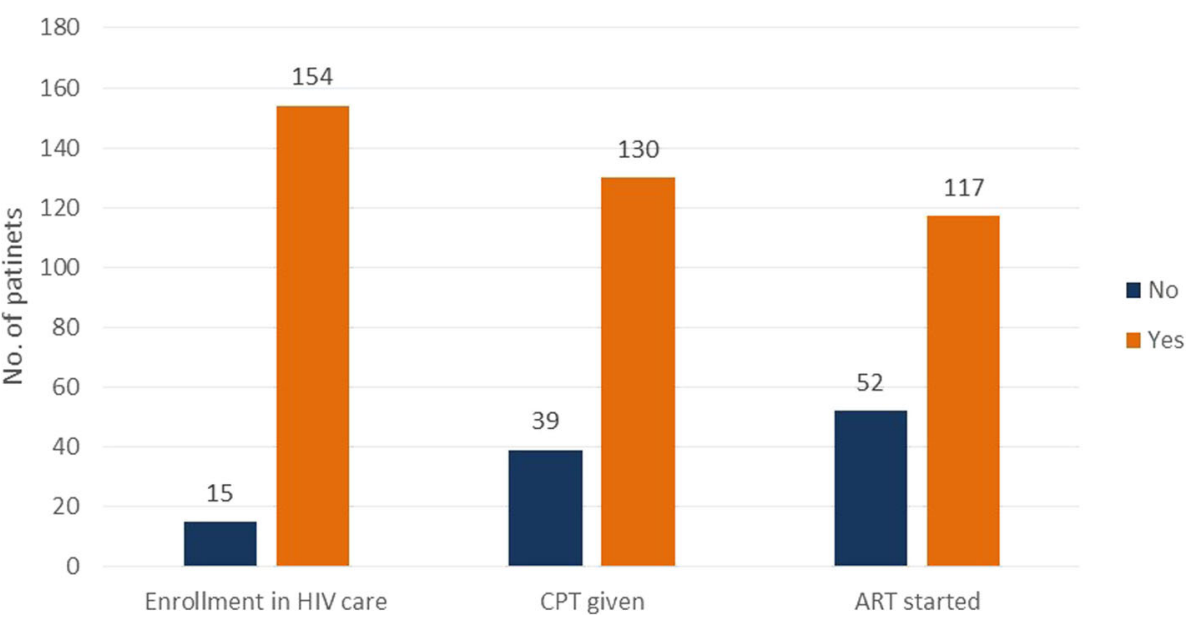

Fig. 1 Status of HIV co-infected study participants by medical care services received

Looking at HIV co-infected patients separately, the analysis shows that there were 37 patients $(22 \%)$ who had been on ART for more than 6 months when they developed active TB disease. The occurrence of active TB disease in those HIV patients who have been on ART for more than 6 months makes these patients suspected for the possibility of the emergence of treatment failure on the ART regimen that these patients were receiving. This might have dampened the positive effect of ART on TB treatment outcomes. However, a separate analysis comparing the TB treatment outcomes among those who developed active TB disease while taking ART for more than 6 months with the other study participants (who started ART after TB diagnosis or were taking ART less than 6 months when developing active $\mathrm{TB}$ disease) failed to show a significant difference in TB treatment outcome between them $(\mathrm{OR}=0.613$ [0.218-1.726]; $P=0.354)$.

For those factors that showed significant association on initial analysis, multiple regression analysis was done. The results show that these factors are still significantly associated with outcomes of TB treatment (Table 5).

\section{Discussion}

In this study, more than half (58.3 \%) of the study participants were in the age groups between 25 and 49 years. Among the 575 study participants who were enrolled into the study sample, 529 (92\%) have a documented HIV test result, among whom about one-third (1/3) were HIV-positive. These findings are much higher than that reported for Ethiopia for 2013 with just $71 \%$ of TB patients having a documented HIV

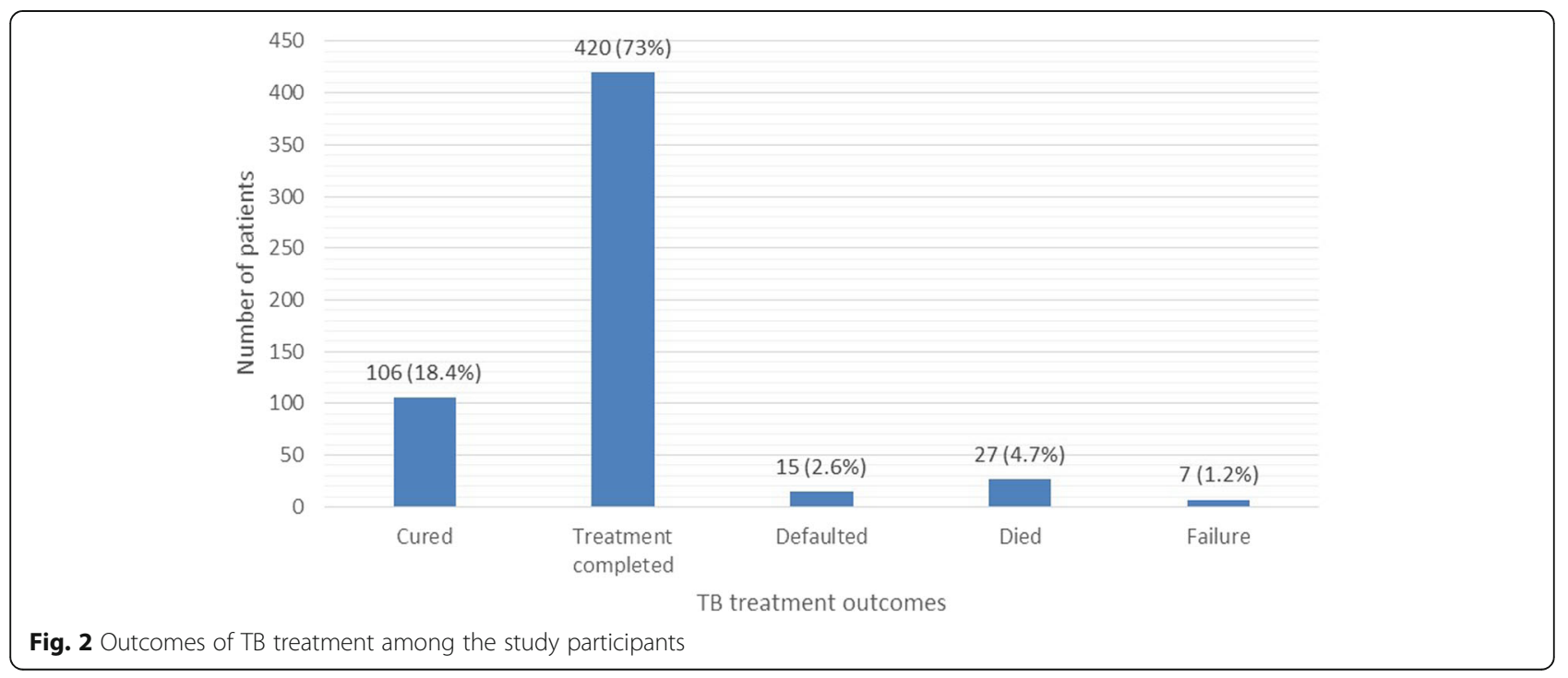


Table 2 Comparison of TB treatment success based on HIV status $(N=529)$

\begin{tabular}{llll}
\hline TB treatment outcome & \multicolumn{2}{l}{ HIV status } & Total \\
\cline { 2 - 3 } & HIV negative & HIV positive & \\
\hline Unfavourable outcome & $23(6.4 \%)$ & $20(11.8 \%)$ & $43(8.1 \%)$ \\
$\begin{array}{llll}\text { Favourable outcome } \\
\text { (treatment success) }\end{array}$ & $337(93.6 \%)$ & $149(88.2 \%)$ & $486(91.9 \%)$ \\
Total & $360(100.0 \%)$ & $169(100.0 \%)$ & $529(100.0 \%)$ \\
\hline
\end{tabular}

status and only $11 \%$ of those with documented status being HIV-positive [13].

Pulmonary tuberculosis was the most common presentation of TB disease among the study participants $(55.8 \%)$, and a quarter of all cases of TB were found to have smear-positive pulmonary tuberculosis (P/pos). Extra-Pulmonary (EP) cases accounted for $44 \%$ of the total.

Among those study participants who had coinfection with HIV $(N=169)$, a high proportion of them were receiving care and treatment services for HIV with 91.1 \% enrolled in HIV care, 76.9 \% received co-trimoxazole prophylactic treatment and $69.2 \%$ regularly receiving ART.

There was a very high rate of treatment success observed in this study. Overall, treatment success rate was $91.5 \%$ for all the study participants. The success rate was $88.2 \%$ among HIV co-infected TB patients in contrast to $93.6 \%$ for all non-HIV infected TB patients. HIV co-infected TB patients have a higher rate (11.8\%) of unfavourable treatment outcomes (defined as death, default or failure) compared to those without underlying HIV infection (6.4\%). Other similar studies conducted in high prevalence settings showed that the highest treatment success rate (85\%) is observed in HIV noninfected TB patients and overall success rate (for both groups combined) is $77 \%$ ([14]:521-528; [15]:157). From global TB reports by the WHO, the best treatment success rates (95\%) were reported from China among all new TB patients [13]. The reported treatment success rate for Ethiopia among all new TB cases in 2012 was $91 \%$, which is the highest reported yet for this country and showing a progressively improving trend over the years [13]. The same report indicates that the treatment success rate for Ethiopia was less than $80 \%$ in the years prior to the year 2011 .

The cure rate among HIV co-infected TB patients was significantly lower than among those without underlying HIV infection (10.1\% versus $24.2 \%$ ); whereas, the death rate was higher in HIV co-infected TB patients $(8.3 \%$ versus $2.5 \%$ ). These findings are consistent with the expected outcomes among HIV co-infected patients including observations from studies and programme reports. According to the global TB report [13], globally in 2012, HIV co-infected TB patients had $74 \%$ favourable treatment outcomes compared with $88 \%$ for HIVnegative TB patients, though the difference was smaller in the African region (75\% in co-infected versus $83 \%$ in non-HIV infected). In this same report, there were very similar findings regarding TB patient mortality which showed the proportion of TB patients that died during treatment was more than three times higher among HIV-co-infected TB patients (11\% versus $3.4 \%$ ).

In addition to HIV status, age and TB classification were significantly associated with TB treatment outcome in this study. TB patients in the age group above 65 years of age had a higher likelihood of "unfavourable outcome" than those in the other age groups. Other studies have also demonstrated the association of adverse TB treatment outcomes and advanced age. A study conducted in South India ([16]:e67288) showed that TB treatment outcomes were poor among older TB patients

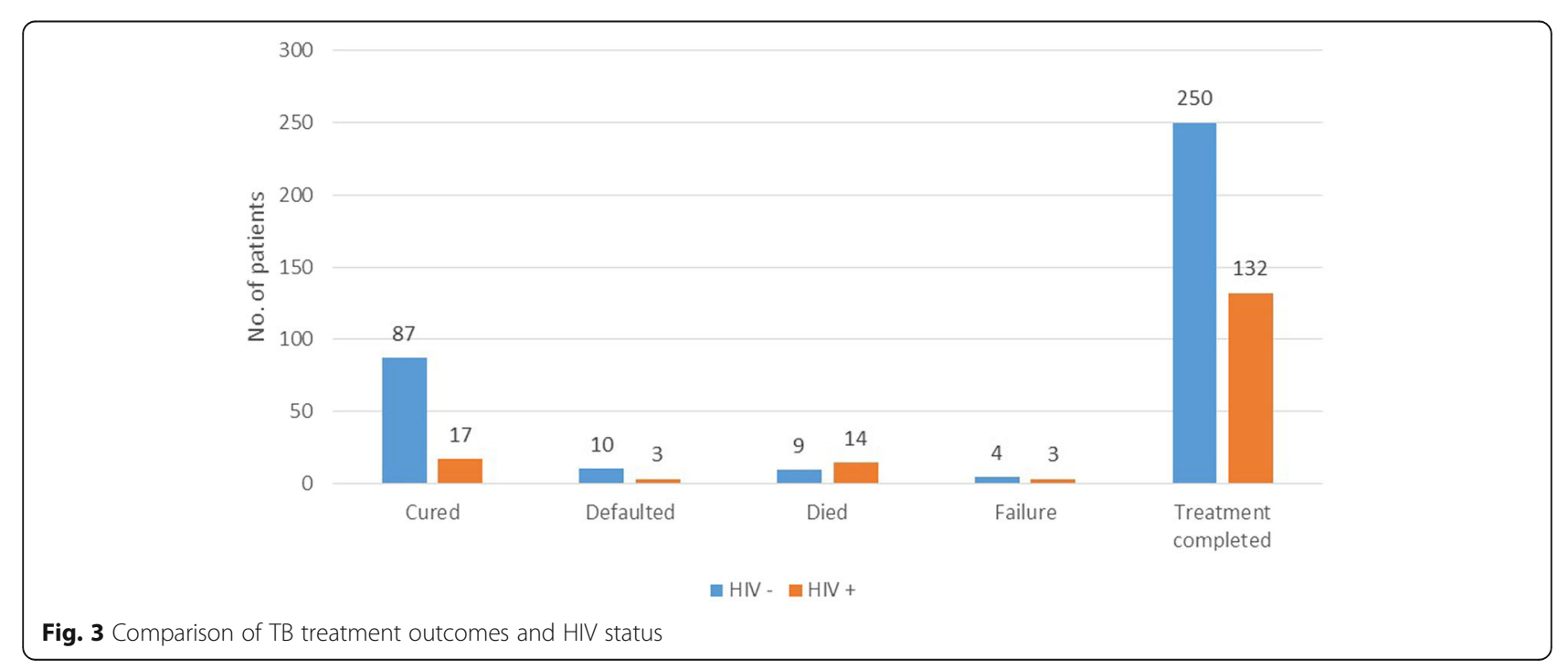


Table 3 Comparison of TB treatment outcomes based on HIV status ( $N=529)$

\begin{tabular}{|c|c|c|c|c|c|}
\hline Treatment outcome & HIV - & $\mathrm{HIV}+$ & OR & $95 \% \mathrm{Cl}$ & $P$ value \\
\hline Cured & 87 (24.2\%) & 17 (10.1\%) & 2.702 & $1.542-4.735$ & 0.001 \\
\hline Defaulted & $10(2.8 \%)$ & $3(1.8 \%)$ & 1.76 & $0.476-6.505$ & 0.397 \\
\hline Died & $9(2.5 \%)$ & $14(8.3 \%)$ & 0.339 & $0.143-0.805$ & 0.014 \\
\hline Failure & $4(1.1 \%)$ & $3(1.8 \%)$ & 0.704 & $0.155-3.192$ & 0.649 \\
\hline Treatment completed & $250(69.4 \%)$ & $132(78.1 \%)$ & & & \\
\hline Total & $360(100 \%)$ & $169(100 \%)$ & & & \\
\hline
\end{tabular}

and there was an increased risk of unfavourable outcomes in those above the age of 60 years. Another study in Delhi, India also showed a significantly higher rate of death and TB treatment failure among TB patients older than 65 years of age ([17]:83).

Lower TB treatment success was observed among those patients with smear positive pulmonary TB. This is consistent with the results of other studies in similar settings. A study in India demonstrated that TB disease classification is significantly associated with TB treatment outcome and patients under 'Pulmonary' TB classification had "unfavourable outcome" ([18]:e21008). Another Nigerian study showed that those TB patients with smear positive status at diagnosis (i.e. pulmonary positive TB classification) have more adverse TB treatment outcomes ([19]:210).

The unexpected finding in this study was the absence of association between TB treatment outcome and the other medical care services received by HIV co-infected TB patients, namely starting ART, Cotrimoxazole prophylactic treatment and enrolment in HIV care. These findings are in contrast to results of other studies from other settings which showed that TB patients who were put on ART had more successful treatment outcomes than those not taking ART ([20]:e56248; [18]:e21008). A similar study in HIV co-infected TB patients in Italy showed that there is a marked reduction in death rate in patients who were taking ART during tuberculosis treatment; but patients who were already on ART when they were diagnosed to have tuberculosis had higher risk of death ([21]:1).

Additional analysis of the data from our study showed that $22 \%$ of HIV co-infected TB patients were taking ART for more than 6 months when they were diagnosed to have active TB disease and started on TB treatment. This finding could be indicative of the emergence of

Table 4 Binary regression analysis of the association of patients' characteristics with TB treatment outcome

\begin{tabular}{|c|c|c|c|c|c|c|c|}
\hline \multirow[t]{2}{*}{ Variable } & \multirow[t]{2}{*}{ Value } & \multicolumn{2}{|c|}{ TB treatment outcome } & \multirow[t]{2}{*}{ Total } & \multirow[t]{2}{*}{ OR } & \multirow[t]{2}{*}{$95 \% \mathrm{Cl}$} & \multirow[t]{2}{*}{$P$ value } \\
\hline & & No success & Success & & & & \\
\hline \multirow[t]{5}{*}{ Age } & $15-24$ & $9(6.0 \%)$ & $142(94.0 \%)$ & 151 & 0.243 & $0.079-0.747$ & 0.014 \\
\hline & $25-34$ & $15(8.2 \%)$ & $169(91.8 \%)$ & 184 & 0.34 & $0.120-0.965$ & 0.043 \\
\hline & $35-49$ & $10(6.6 \%)$ & $141(93.4 \%)$ & 151 & 0.272 & $0.090-0.820$ & 0.021 \\
\hline & $50-64$ & $9(15 \%)$ & $51(85 \%)$ & 60 & 0.676 & $0.215-2.124$ & 0.503 \\
\hline & $65-90$ & $6(20.7 \%)$ & $23(79.3 \%)$ & 29 & & & \\
\hline \multirow[t]{2}{*}{ Gender } & $\mathrm{F}$ & $22(8.3 \%)$ & $242(91.7 \%)$ & 264 & 0.956 & $0.531-1.722$ & 0.881 \\
\hline & M & $27(8.7 \%)$ & $284(91.3 \%)$ & 311 & & & \\
\hline \multirow[t]{3}{*}{ TB classification } & EP & 17 (6.7 \%) & $236(93.3 \%)$ & 253 & 0.436 & $0.22-0.863$ & 0.017 \\
\hline & P/neg & $12(6.7 \%)$ & $168(93.3 \%)$ & 180 & 0.432 & $0.204-0.917$ & 0.029 \\
\hline & P/pos & 20 (14.2\%) & $121(85.8 \%)$ & 141 & & & \\
\hline \multirow[t]{2}{*}{ Enrollment in HIV care } & No & $3(20.0 \%)$ & 12 (80.0 \%) & 15 & 2.015 & $0.516-7.864$ & 0.313 \\
\hline & Yes & $17(11.0 \%)$ & 137 (89 \%) & 154 & & & \\
\hline \multirow[t]{2}{*}{ Co-trimoxazole given } & No & 7 (17.9\%) & 32 (82.1\%) & 39 & 1.969 & $0.725-5.344$ & 0.184 \\
\hline & Yes & $13(10.0 \%)$ & $117(90.0 \%)$ & 130 & & & \\
\hline \multirow[t]{2}{*}{ ART started } & No & 8 (15.4 \%) & 44 (84.6 \%) & 52 & 1.591 & $0.608-4.161$ & 0.344 \\
\hline & Yes & 12 (10.3\%) & $105(89.7 \%)$ & 117 & & & \\
\hline \multirow[t]{2}{*}{ HIV status } & Negative & 23 (6.4 \%) & 337 (93.6 \%) & 360 & 0.508 & $0.271-0.954$ & 0.035 \\
\hline & Positive & 20 (11.8\%) & 149 (88.2 \%) & 169 & & & \\
\hline
\end{tabular}


Table 5 Multiple regression analysis of factors associated with TB treatment outcome

\begin{tabular}{|c|c|c|c|c|c|c|c|c|}
\hline \multirow[t]{2}{*}{ Variable } & \multirow[t]{2}{*}{ Value } & \multicolumn{2}{|c|}{ TB treatment outcome } & \multirow[t]{2}{*}{ Total } & \multirow[t]{2}{*}{ OR } & \multirow[t]{2}{*}{ AOR } & \multirow[t]{2}{*}{$95 \% \mathrm{Cl}$} & \multirow{2}{*}{$\begin{array}{l}P \\
\text { value }\end{array}$} \\
\hline & & No success & Success & & & & & \\
\hline \multirow[t]{5}{*}{ Age } & $15-24$ & $9(6.0 \%)$ & $142(94.0 \%)$ & 151 & 0.243 & 0.150 & $0.041-0.546$ & 0.004 \\
\hline & $25-34$ & 15 (8.2 \%) & 169 (91.8 \%) & 184 & 0.34 & 0.185 & $0.055-0.619$ & 0.006 \\
\hline & $35-49$ & $10(6.6 \%)$ & 141 (93.4 \%) & 151 & 0.272 & 0.126 & $0.034-0.468$ & 0.002 \\
\hline & $50-64$ & $9(15 \%)$ & $51(85 \%)$ & 60 & 0.676 & 0.389 & $0.104-1.457$ & 0.161 \\
\hline & $65-90$ & $6(20.7 \%)$ & $23(79.3 \%)$ & 29 & & & & \\
\hline \multirow[t]{3}{*}{ TB classification } & EP & $17(6.7 \%)$ & $236(93.3 \%)$ & 253 & 0.436 & 0.322 & $0.149-0.697$ & 0.004 \\
\hline & P/neg & 12 (6.7 \%) & 168 (93.3 \%) & 180 & 0.432 & 0.329 & $0.146-0.744$ & 0.008 \\
\hline & P/pos & $20(14.2 \%)$ & $121(85.8 \%)$ & 141 & & & & \\
\hline \multirow[t]{2}{*}{ HIV status } & Negative & $23(6.4 \%)$ & 337 (93.6 \%) & 360 & 0.508 & 0.367 & $0.178-0.757$ & 0.007 \\
\hline & Positive & $20(11.8 \%)$ & $149(88.2 \%)$ & 169 & & & & \\
\hline
\end{tabular}

treatment failure on the ART regimen that these patients were taking. This could have contributed to dampen the positive effect of ART on TB treatment outcomes. However, no difference in treatment outcome was observed between those who developed active TB disease while taking ART for more than 6 months and the other study participants. Another point to consider would be that many HIV co-infected TB patients might have been diagnosed too late in the course of their illnesses (advanced stages of HIV) to have any significant benefit from HIV care and treatment services.

\section{Limitations}

A limitation of this study could be that all the sites are within Addis Ababa which is the capital and the largest town in the country. Patients from these facilities might have a different profile from patients residing in other parts of the country.

Another limitation is that the data collected is retrospective secondary data. For some study participants, there were issues with missing and/or inaccurate data. In addition, the data source (which was the standard TB registers) did not capture detailed information on HIV co-infected TB patients' clinical and immunological status (ex. CD4 count, viral load), and other HIV related services (ex. ARV regimens, adherence).

Prospective quantitative studies and qualitative studies are required to further explore additional patient level and service delivery factors that may affect the outcome of HIV co-infected TB patients.

\section{Conclusions}

In this study, we observed that there was a very high rate of TB treatment success, even among HIV co-infected TB patients. Nevertheless, there were more unfavourable outcomes among the HIV co-infected TB patients, and the provision of HIV treatment and care services (ART, co-trimoxazole etc.) was not found to have significant effect on TB treatment outcome. Further prospective and qualitative studies will be important to assess the reason why receiving HIV care and treatment services had been seen to have no effect on TB treatment outcomes of HIV co-infected TB patients. Delayed identification of HIV and/or TB might have negatively affected the outcomes of these patients. In addition, it is worthwhile to investigate individual patient level factors and/ or treatment regimen related factors for TB patients with advanced age and those with smear positive pulmonary $\mathrm{TB}$, as more unfavourable outcomes were observed among these patients.

\section{Additional file}

Additional file 1: Cleaned data on Outcomes of TB treatment Ethiopia. This is the cleaned set of data of the 575 individual study participants that were included in the analysis. It shows the age and gender of the participants, their clinical characteristics and medical care received, and their individual health outcomes. (XLSX $55 \mathrm{~kb}$ )

\section{Abbreviations}

ART: Anti-Retroviral Treatment; EP: Extra pulmonary tuberculosis; HIV: Human Immunodeficiency Virus; P/pos: Smear Positive Pulmonary Tuberculosis; SSA: sub-Saharan Africa; TB: Tuberculosis; UCSF: University of California San Francisco; UNISA: University of South Africa; WHO: World Health Organization;

\section{Acknowledgements}

The following individuals and institutions helped significantly in conducting this study either by reviewing, editing or facilitating access to the health facilities and data sources.

Dr. Thomas Heller, Associate Director for Care and Treatment, CDC-Ethiopia. Addis Ababa City Administration Health Bureau, Ethiopia.

The heads and health workers of the health facilities in Addis Ketema health center, Bole 17 health Center, Kazanchis health center, Kotebe health center, Semien health center, Shiromeda health center, Wereda 9 (Nifas Silk) health center, and Yeka health center.

Mrs. Engela Catharina Coetzer, Department of Health Studies, UNISA, South Africa.

Funding

No funding from any source was obtained for this study. 


\section{Availability of data and materials}

The dataset(s) supporting the conclusions of this article is (are) included within the article (and its additional file(s)).

\section{Authors' contributions}

The authors of these manuscript are SA, TM, RF and TA. SA is the lead investigator of the study. He initiated the study by identifying the problem statement, selecting the study title, and designing, developing and finalizing the study protocol. He secured approval and ethical clearance from the local IRB and the UNISA. He conducted the study in data collection, cleaning and analysis. He worked in the entire write up, revision and submission of the manuscript. TM was involved in all stages of the development of the study protocol making substantial contributions in the conception and design of the study by reviewing, couching, and guiding. He contributed in finalizing and approval of the study protocol. He had substantial contribution in the analysis, interpreting and write up of the data. He made significant inputs to the write-up of the manuscript of the study in the dissertation. RF was involved in the conception and design of the study. She worked in supporting the developing and finalizing of the study protocol. She made significant contributions in data collection tool development, data collection and cleaning, and analysis and interpreting the results. She made substantial contributions in writing-up and revision of the manuscript. TA made substantial contributions in developing and validating data collection tool, data collection, and analysis and interpretation of data, particularly statistical tests on the collected data. He was heavily involved in revising and structuring the manuscript in intellectual content and suitability for publication. All authors read and approved the final manuscript.

\section{Competing interests}

The authors declare that they have no competing interests.

\section{Consent for publication}

Not applicable.

\section{Ethics approval and consent to participate}

Prior to the commencement of the study, the final version of the study protocol had been submitted and ethical clearance obtained from the local IRB (Addis Ababa Regional Health Bureau Ethical Review Committee) and the UNISA College of Human Sciences (CHS) Health Studies Higher Degrees Committee (HSHDC).

The data collected was secondary data from TB clinics register and the need for consent to participate was waived. The waiver was provided or approved by UNISA CHS HSHDC and the local IRB after the study protocol was submitted and reviewed for ethical clearance before data collection.

\section{Author details}

'Department of Health Studies, University of South Africa (UNISA), Pretoria, South Africa. ${ }^{2}$ Addis Continental Institute of Public Health, Addis Ababa, Ethiopia. ${ }^{3}$ University of Gondar, Gondar, Ethiopia. ${ }^{4}$ Addis Ababa, Ethiopia.

\section{Received: 30 March 2016 Accepted: 25 October 2016}

\section{Published online: 04 November 2016}

\section{References}

1. Getahun H, Gunneberg C, Granich R, Nunn P. HIV infection-associated tuberculosis: the epidemiology and the response. Clin Infect Dis. 2010; 50(S3):S201-7. http://cid.oxfordjournals.org/content/50/Supplement_3/S201. full.pdf+html. Accessed 17 Apr 2014.

2. World Health Organization. Global Tuberculosis Report. 2012. http:// apps.who.int/iris/bitstream/10665/75938/1/9789241564502_eng.pdf. Accessed 12 Jun 2013.

3. Friedland G, Churchyard GJ, Nardell E. Tuberculosis and HIV coinfection: current state of knowledge and research priorities. J Infect Dis. 2007; 196(Supplement 1):S1-3.

4. World Health Organization. WHO policy on collaborative TB/HIV activities. Guidelines for national programmes and other stakeholders. 2012. http://whqlibdoc.who.int/publications/2012/9789241503006_eng.pdf. Accessed 20 May 2013.

5. Daniel OJ, Alausa OK. Treatment outcome of TB/HIV positive and TB/HIV negative patients on directly observed treatment, short course (DOTS) in Sagamu, Nigeria. NigerJ Med. 2006;15(3):222-6.
6. World Health Organization. WHO Report Global Tuberculosis Control. 2011. http://whqlibdoc.who.int/publications/2011/9789241564380_eng.pdf. Accessed 07 Jun 2013.

7. Lawn SD, Myer L, Bekker LG, Wood R. Burden of tuberculosis in an antiretroviral treatment programme in sub-Saharan Africa: impact on treatment outcomes and implications for tuberculosis control. AIDS. 2006;20(12):1605-12.

8. Ethiopian Health and Nutrition Research Institute, Federal Ministry of Health HIV related estimates and projections for Ethiopia. 2012. http://files.unaids. org/en/media/unaids/contentassets/documents/data-and-analysis/tools/ spectrum/Ethiopia2012report.pdf.

9. University of California San Francisco. Comprehensive, up-to-date information on HIV/AIDS treatment, prevention, and policy. 2013. http://hivinsite.ucsf.edu/InSite?page=kb-05-01-06. Accessed 14 Oct 2013.

10. Federal Ministry of Health, Ethiopia. Tuberculosis, leprosy and TB/HIV prevention and control programme manual. 4th ed. Ethiopia: Federal Ministry of Health; 2008.

11. Federal Democratic Republic of Ethiopia Ministry of Health. Guidelines for clinical and programmatic management of TB, Leprosy and TB/HIV in Ethiopia. 5th edn. Addis Ababa; 2013.

12. World Health Organization. Treatment of tuberculosis Guidelines. 4th edition 2010. http://whqlibdoc.who.int/publications/2010/9789241547833_eng.pdf. Accessed 03 Jun 2013.

13. World Health Organization. Global Tuberculosis Report. 2014. http://apps. who.int/iris/bitstream/10665/137094/1/9789241564809_eng.pdf?ua=1. Accessed 25 Oct 2014

14. Tripathy S, Anand A, Inamdar V, Manoj MM, Khillare KM, Datye AS, Iyer R, Kanoj DM, Thakar M, Kale V, Pereira M, Risbud AR. Clinical response of newly diagnosed HIV seropositive \& seronegative pulmonary tuberculosis patients with the RNTCP short course regimen in Pune, India. Indian J Med Res. 2011;133(5):521-8.

15. Sharma SK, Soneja M, Prasad KT, Ranjan S. Clinical profile and predictors of poor outcome of adult HIV-tuberculosis patients in a tertiary care centre In North India. Indian J Med Res. 2014;139:154-60.

16. Ananthakrishnan R, Kumar K, Ganesh M, Kumar AM, Krishnan N, Swaminathan S, Edginton MKA, Gupta D. The profile and treatment outcomes of the older (aged 60 years and above) tuberculosis patients in Tamilnadu, South India. PLoS One. 2013;8(7):e67288

17. Gaur SN, Dhingra VK, Rajpal S, Aggarwal JK, Meghna. Tuberculosis in the elderly and their treatment outcome under DOTS. Indian J Tuberc. 2004;51: $83-7$.

18. Vijay S, Kumar P, Chauhan LS, Narayan Rao SV, Vaidyanathan P. Treatment outcome and mortality at one and half year follow-up of HIV infected TB patients under TB control programme in a district of South India. PLOS ONE. 2011;6(7):e21008.

19. Babatunde $\mathrm{OA}$, Elegbede $\mathrm{OE}$, Ayodele M, Fadare JO, Isinjaye AO, Ibirongbe DO, Akinyandenu J. Factors affecting treatment outcomes of tuberculosis in a Tertiary Health Center in Southwestern Nigeria. Int Rev Soc Sci Humanit. 2013;4(2):209-18.

20. Tweya H, Feldacker C, Phiri S, Ben-Smith A, Fenner L, Jahn A, Kalulu M, Weigel R, Kamba C, Banda R, Egger M, Keiser O. Comparison of treatment outcomes of new smear-positive pulmonary tuberculosis patients by HIV and antiretroviral status in a TB/HIV clinic, Malawi. PLoS ONE. 2013;8(2):e56248.

21. Girardi E, Palmieri F, Angeletti C, Vanacore P, Matteelli A, Gori A, Carbonara S, Ippolito G. Impact of previous ART and of ART initiation on outcome of HIVassociated tuberculosis. Clin Dev Immunol. 2012;2012:1-8. 